\title{
Up-regulated miR-106b inhibits ox-LDL-induced endothelial cell apoptosis in atherosclerosis
}

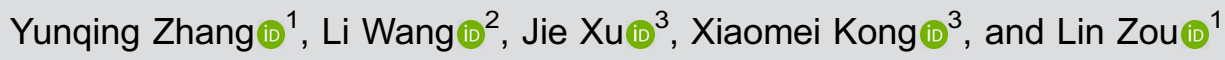 \\ ${ }^{1}$ Department of Cardiology, Zuanshiwan Branch of The Second Affiliated Hospital of Dalian Medical University, \\ Dalian, Liaoning, China \\ ${ }^{2}$ Department III of Cardiology, The Central Hospital of Dalian, Dalian, Liaoning, China \\ ${ }^{3}$ Department of Endocrinology, Zuanshiwan Branch of The Second Affiliated Hospital of Dalian Medical University, \\ Dalian, Liaoning, China
}

\begin{abstract}
This research aimed to explore the molecular mechanism of microRNA (miR)-106b in cell apoptosis of atherosclerosis (AS). Human aortic endothelial cells (HAECs) were divided into control group, oxidized-low-density lipoproteins (ox-LDL) group, miR106b NC + ox-LDL group, miR-106b mimics + ox-LDL group, miR-106b mimics + PTEN + ox-LDL group, and miR-106b mimics + empty + ox-LDL group. Real-time fluorescence quantitative polymerase chain reaction, cholecystokinin, TdT-mediated biotinylated nick end-labeling assay, luciferase reporter gene assay, and flow cytometry analysis were performed to determine the morphology, proliferation, and apoptosis in HSECs. Moreover, the levels of phosphatase and tensin homolog deleted on chromosome 10 (PTEN), Bcl-2, p-P13K, and p-AKT in HAECs were detected by western blot. MiR-106b was down-regulated in ox-LDL-induced HAECs. PTEN was the target gene of miR-106b-5p. Overexpression of PTEN inhibited the anti-apoptotic effect of miR-106b. Compared with the control group, the proportion and number of HAECs apoptosis and Bax, caspase-3, and caspase-9 expression in ox-LDL and miR-106b mimics + PTEN + ox-LDL groups were significantly increased (all $P<0.05$ ). Moreover, the activity of HAECs and Bcl-2 were decreased significantly (all $P<0.05$ ). Overexpression of miR-106b in ox-LDLinduced AS inhibited endothelial cell apoptosis. Furthermore, miR-106b might activate the PI3K/AKT pathway by downregulating the expression of PTEN in ox-LDL-induced HAECs.
\end{abstract}

Key words: Atherosclerosis; ox-LDL; miR-106b; PTEN; P13K/AKT; Endothelial cell apoptosis

\section{Introduction}

Atherosclerosis (AS) is a chronic inflammatory process of the arterial wall caused by hyperlipidemia (1). There are two prominent hallmarks of AS pathogenesis including the accumulation of cholesterol in the endothelial lining of arteries carried by low-density lipoproteins (LDL) and chronic inflammation due to a high ratio of prooxidants to antioxidants $(2,3)$. As a systemic disease, AS and its accompanying clinical complications are important factors in long-term mortality and morbidity worldwide $(4,5)$. Although various strategies such as drug therapy and surgery have been used for the clinical treatment of AS, the outcome of AS treatment is still not optimal due to the lack of a deep understanding of the pathological mechanism of AS $(6,7)$.

MicroRNAs (miRNAs) act as significant regulators in the pathophysiology of AS (8). As a member of miRNAs, the miR-106b family is associated with the level of genes regulating the cell cycle, which promotes cancer cell proliferation by shortening cell cycle progression (9). The differential expression of miR-106b has been proven to take part in the development of various diseases such as prostate cancer, lung cancer, and gastric cancer (10-12). A previous study shows that the abnormal expression of miR-106b is closely related with AS progression (13).

The biological function of miR-106b in disease can be via certain pathways (14). Yan et al. (15) showed that drugs could inhibit inflammation and promote the stability of AS plaques by regulating the PI3K/Akt pathway. Phosphatase and tensin homolog deleted on chromosome 10 (PTEN) is a viable target for the prevention of apoptosis in vascular endothelial cells $(16,17)$. A previous study indicated that miR-106b promotes pituitary tumor cell proliferation and invasion through PI3K/AKT signaling pathway by targeting PTEN (18). Shi et al. (19) reported that miR-106b-5p participates in the PI3K/AKT pathway by regulating $\mathrm{PTEN}$, thereby promoting stem cell-like properties in liver cancer cells. Furthermore, human aortic endothelial cells (HAECs) are commonly used for the

Correspondence: Lin Zou: <zoulin2019@163.com> 
pathological study of AS since cytokine secretion by HAECs is related to the degree of AS (20). Previous study indicates that miR-21 suppresses oxidized-LDL (ox-LDL)induced HAECs injuries in AS (21). Although the function of miRNA-106b has been reported in the development of various diseases, the relationship between miR-106b and AS is still not clear.

In this study, the mechanism of miR-106b in AS was explored based on ox-LDL-treated HAECs. Biological function of miR-106b in ox-LDL-treated HAECs progression was investigated. Our findings suggested that miR106b exerted a pro-proliferation role in ox-LDL-treated HAECs and could activate the PI3K/AKT pathway.

\section{Material and Methods}

\section{Cell culture and transfection}

HAECs were cultured in endothelial cell culture medium containing $1 \%$ endothelial growth factor and $5 \%$ fetal bovine serum (FBS) at $37^{\circ} \mathrm{C}\left(5 \% \mathrm{CO}_{2}\right)$. HAECs were transfected with miR-106b NC, miR-106b mimics, PTEN expression plasmid, or empty plasmid (Shanghai Gemma Gene, China) by Lipofectamine 2000 for $24 \mathrm{~h}$, followed by ox-LDL (50 $\mu \mathrm{g} / \mathrm{mL}$, Beijing Xiesheng Biotechnology Co., Ltd., China) treatment for $24 \mathrm{~h}$. Then, all cells were divided into ox-LDL group, miR-106b NC+ox-LDL group, miR-106b mimics + ox-LDL group, miR-106b mimics + PTEN + ox-LDL group, and miR-106b mimics + empty + ox-LDL group. Cells without ox-LDL treatment were considered as the control group.

\section{CCK-8 assay}

Cell activity was detected by CCK-8 assay. Briefly, HAECs were seeded on a 96-well plate $\left(1 \times 10^{4}\right.$ cells/well $)$ with a concentration of $10 \mu \mathrm{L} /$ well CCK-8 solution (Engreen Biosystem, China). Plates were incubated at $37^{\circ} \mathrm{C}$ for $2 \mathrm{~h}$, and absorbance at $450 \mathrm{~nm}$ was recorded by a microplate reader (Bio-Rad, USA).

\section{Flow cytometry assay}

Flow cytometry was used for HAECs apoptosis detection. Briefly, after re-suspended with $100 \mu \mathrm{L}$ PBS, cells from each group were suspended with $5 \mu \mathrm{L}$ FITC-AnnexinV $(1 \mu \mathrm{g} / \mathrm{mL})$ and $5 \mu \mathrm{L} \mathrm{PI}(1 \mu \mathrm{g} / \mathrm{mL})$. Then, all cells were quantitatively detected by FACScan flow cytometry (BD Biosciences, USA) with CellQuest software (BD Biosciences).

\section{TdT-mediated biotinylated nick end-labeling (TUNEL) assay}

TUNEL assay was performed to detect the HAECs cell apoptosis from each group. According to the TUNEL kit (Beyotime Biotechnology, China) instructions, the HAECs were fixed with $4 \%$ formaldehyde and then permeated with $0.1 \%$ Triton $\mathrm{X}-100$. Next, the cells were cultured with the mixture of TUNEL reaction at $37^{\circ} \mathrm{C}$ for $1 \mathrm{~h}$. Finally, HAECs were observed under a laser confocal microscope (FV300, Olympus, Japan).

\section{RT-qPCR}

The expression of miR-106b was detected using RTqPCR (ABI7500 (Thermo Fisher Scientific, USA). Total RNAs were extracted from cells using TRizol reagent (Invitrogen, USA), and reverse transcription was performed using Takara reverse transcription kit (Japan). The amplification primer sequence of each gene is listed in Table 1. In addition, U6 was the internal reference. The data were analyzed by the $2^{-\Delta \Delta \mathrm{Ct}}$ method (22).

\section{Western blot assay}

The expression of proteins in HAECs was detected by western blot. Total protein of HAECs from different groups were extracted by RIPA lysis buffer (Beyotime Biotechnology) containing protease and phosphatase inhibitors (complete ULTRA Tablets, Roche, USA). After centrifugation at $12,000 \mathrm{~g}$ for $5 \mathrm{~min}$ at $4^{\circ} \mathrm{C}$, proteins were separated by $10 \%$ SDS-polyacrylamide gel electrophoresis and transferred to a polyvinylidenefluoride membrane (Roche). The membrane was then blocked with $50 \mathrm{~g} / \mathrm{L}$ skim milk for $12 \mathrm{~h}$ at $4^{\circ} \mathrm{C}$, and incubated with primary antibodies: GAPDH, $\beta$ actin, PTEN, Bcl-2, Bax, caspase-3, caspase-9, p-P13K, P13K, p-AKT, and AKT (1:1000; Cell Signaling Technology, USA) overnight at $4^{\circ} \mathrm{C}$. Then, the membrane was incubated with secondary antibody (HRP-conjugated anti-mouse IgG, 1:2000, Beijing Boaosen Biotechnology Co., Ltd., China) for $2 \mathrm{~h}$ at $37^{\circ} \mathrm{C}$. Protein bands were visualized using Epson photo 1650 (Seiko Epson Corporation, Japan). Finally, $\beta$-actin was used as an internal reference (1:1000, sc-517582, Santa Cruz Biotechnology, USA), and the relative gray value was analyzed using Quantity One scanning software (Bio-Rad Laboratories, USA).

\section{Luciferase reporter gene assay}

The target sites for miR-106b-5p were determined by Target Scan (www.targetscan.org), and the mutant and wild sequences were designed according to the predicted results. The miR-106b-5p mutant sequence and the wild sequence fragment were cloned and bound to the PGL-3 vector. Then, the 293T cells (ATCC) were seeded onto 24-well plates $\left(5 \times 10^{5}\right.$ cells/well $)$ and co-transformed with a luciferase reporter vector $(0.12 \mu \mathrm{g})$ and $40 \mathrm{nM}$ miR-106b mimic or negative control mimics. After transfection for

Table 1. Primer sequences.

\begin{tabular}{lc}
\hline Primer & Primer sequences \\
\hline U6-F & CTCGCTTCGGCAGCACATATACT \\
U6-R & ACGCTTCACGAATTTGCGTGTC \\
miR-106b-F & CTGGAGTAAAGTGCTGACAGTG \\
miR-106b-R & GTGCAGGGTCCGAGGT \\
PTEN-F & CAAGATGATGTTTGAAACTATTCCAATG \\
PTEN-R & CCTTTAGCTGGCAGACCACAA \\
GAPDH-F & GGAAGGTGAAGGTCGGAGTCA \\
GAPDH-R & GTCATTGATGGCAACAATATCCACT \\
\hline
\end{tabular}


$48 \mathrm{~h}$, the dual luciferase reporter assay kit (Yuanping Hao Biotechnology Co., Ltd., China) was used for evaluation.

\section{Statistical analysis}

All data are reported as means $\pm S D$. Comparisons between groups were performed with Student's $t$-test (two groups) or one-way analysis of variance followed by Fisher's LSD post hoc test (more than two groups). Statistical analysis was performed by Graphpad Prism 5 (Graphpad Software, USA). A P value less than 0.05 was considered to be significantly different.

\section{Results}

\section{MiR-106b was down-regulated in HAECs}

Compared with the control group, the expression of miR-106b in the ox-LDL group decreased significantly $(P=0.014)$. Compared with the miR-106b NC+ox-LDL group, the expression of miR-106b in the mimics + ox-LDL group increased significantly $(P<0.001$, Figure 1$)$.

\section{PTEN was the target gene of miR-106b}

The biological software Targetcsan predicted that the target gene of miR-106b was PTEN (Figure 2A). Moreover, the results of luciferase activity test showed that over-expression of miR-106b significantly decreased the luciferase activity of PTEN-WT-3'UTR, but did not inhibit the luciferase activity of PTEN-MUT-3'UTR (Figure 2B).

\section{MiR-106b inhibited the increase of PTEN in atherosclerosis}

The expression of PTEN mRNA and protein was detected by qRT-PCR (Figure $3 A$ ) and western blot (Figure $3 \mathrm{~B}$ ), respectively. The mRNA and protein levels of HAECs PTEN in the ox-LDL group were significantly

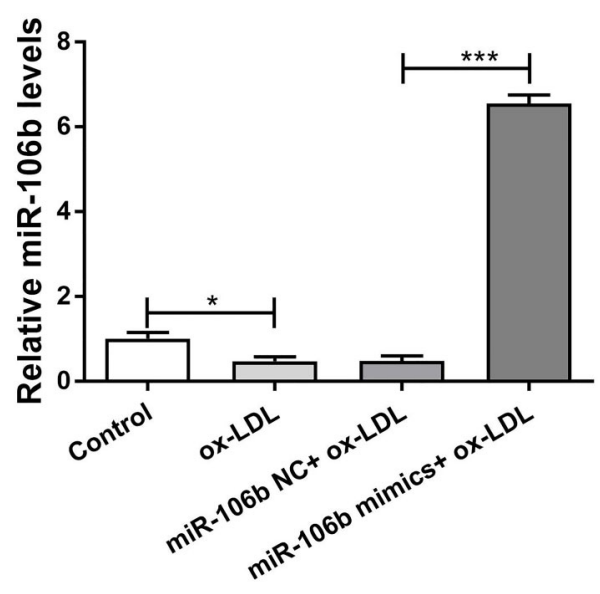

Figure 1. Expression level of microRNA-106b in oxidized-lowdensity lipoproteins (ox-LDL)-induced human aortic endothelial cells. Data are reported as means $\pm S D$. ${ }^{*} P<0.05$, ${ }^{* * *} \mathrm{P}<0.001$ (ANOVA). NC: negative control. higher than those in the control group $(P<0.001)$. Meanwhile, the mRNA and protein levels of HAECs PTEN in the miR-106b mimics + ox-LDL group were significantly lower than those in the miR-106b NC+ox-LDL group $(P<0.001)$. Moreover, the mRNA and protein levels of PTEN in the mir-106b mimics + PTEN + ox-LDL group were significantly higher than those in the mir-106b mimics + empty + ox-LDL group $(P<0.001)$.

\section{Overexpression of miR-106b promoted proliferation and inhibited apoptosis of HAECs}

The proliferation of HAECs was detected by CCK- 8 assay (Figure 4A). The activity of HAECs in the ox-LDL group was significantly lower than that in the control group $(P<0.001)$. The activity of HAECs in the miR-106b mimics +ox-LDL group was significantly higher than that in the miR-106b NC + ox-LDL group $(P<0.001)$. Meanwhile, the activity of HAECs in the miR-106b mimics + PTEN + oxLDL group was significantly lower than that in the miR106b mimics + empty + ox-LDL group $(P<0.001)$.

To further investigate the relationship between HAECs activity and apoptosis, the DNA fragments were detected by TUNEL assay (Figure 4B). The number of TUNELpositive cells in the ox-LDL group was significantly higher than that in the control group $(P<0.001)$. Meanwhile, the number of TUNEL-positive cells in the miR-106b mimics + ox-LDL group was significantly lower than that in the miR-106b NC+ox-LDL group $(P<0.01)$. Moreover, the number of TUNEL-positive cells in the miR-106b mimics + PTEN + ox-LDL group was significantly higher than that in the miR-106b + empty + ox-LDL group $(P<0.001)$. Flow cytometry analysis showed that the apoptotic rate of HAECs in the ox-LDL group was significantly higher than that in the control group $(P<0.001)$ (Figure $4 C)$. The apoptotic rate of HAECs in the miR-106b mimics + ox-LDL group was significantly lower than that in the miR-106b NC+ox-LDL group $(P<0.001)$. The apoptotic rate of HAECs in the miR-106b mimics + PTEN + ox-LDL group was significantly higher than that in the miR-106b mimics + empty + ox-LDL group $(\mathrm{P}<0.001)$. Western blot analysis showed that compared with the control group, the expressions of HAECs pro-apoptotic protein Bax, caspase-3, and caspase- 9 in the ox-LDL group were significantly up-regulated $(P<0.001)$, while the expressions of anti-apoptotic protein bcl-2 were significantly down-regulated $(P<0.001)$ (Figure 4D). The expressions of HAECs pro-apoptotic protein Bax, caspase-3, and caspase- 9 in the miR-106b mimics + ox-LDL group were significantly decreased $(P<0.001)$, while the anti-apoptotic protein bcl2 was significantly up-regulated $(P<0.001)$. Furthermore, compared with miR-106b mimics + empty + ox-LDL group, Bax, caspase- 3 , and caspase- 9 expression were significantly increased $(P<0.001)$, and bcl-2 expression was significantly down-regulated $(P<0.001)$ in the miR-106b mimics + PTEN + ox-LDL group. Taken together, these results suggested that overexpression of miR-106b in 

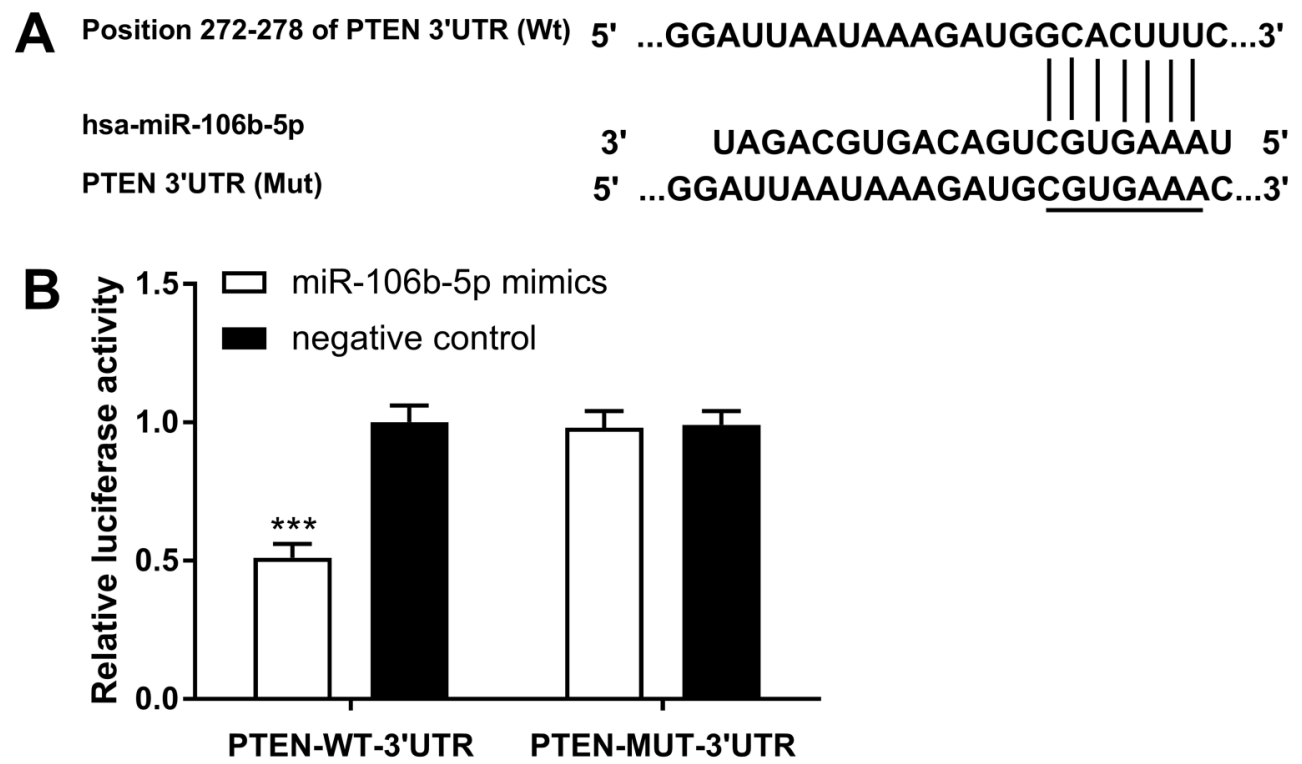

Figure 2. Relationship between PTEN and miR-106b. A, PTEN and miR-106b binding target sites predicted by Target Scan. B, Luciferase activity transfected with indicated luciferase reporters was determined using luciferase report assay. Data are reported as means $\pm \mathrm{SD}$. ${ }^{* * *} \mathrm{P}<0.001$ vs miR-106b mimics and PTEN-MUT-3'UTR co-transfection ( $t$-test).
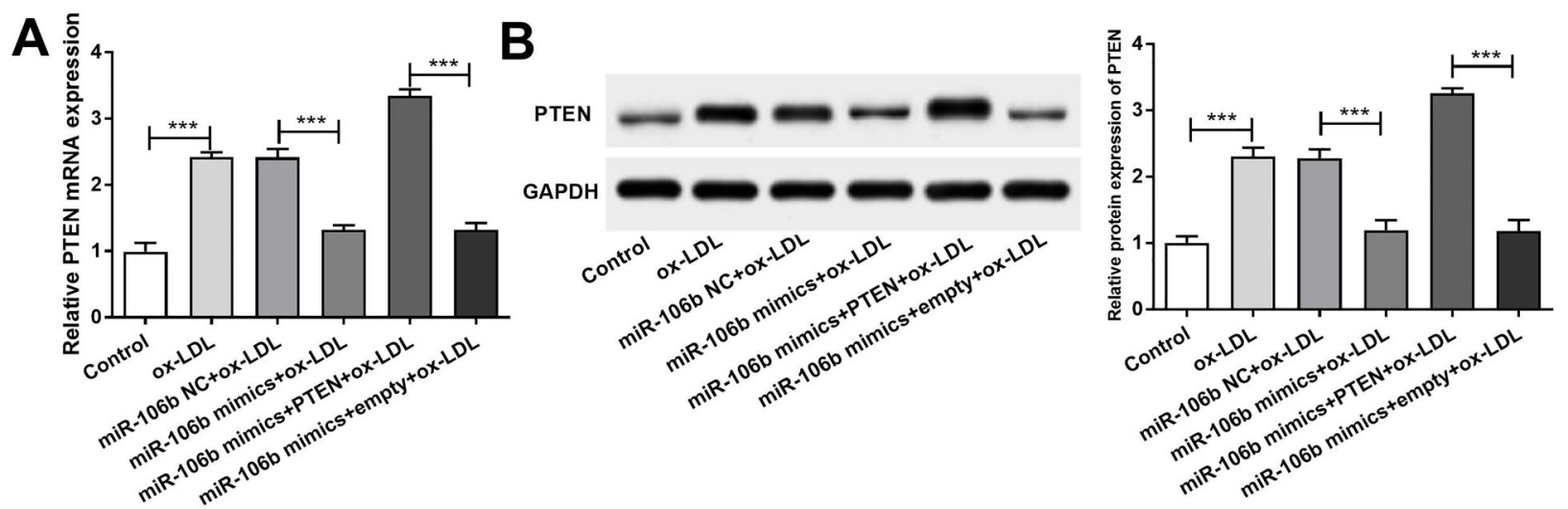

Figure 3. A, mRNA expression level of PTEN in the different groups of cells. B, Protein expression level of PTEN. Data are reported as means $\pm \mathrm{SD}$. ${ }^{* \star} \mathrm{P}<0.001$ (ANOVA). ox-LDL: oxidized-low-density lipoproteins; NC: negative control.

ox-LDL-treated AS could promote HAECs proliferation and inhibit HAECs apoptosis.

\section{MiR-106b targeted PTEN-activated PI3K/Akt signaling pathway in HAECs}

Western blot assay showed that compared with the control group, $\mathrm{p}-\mathrm{P} 13 \mathrm{~K} / \mathrm{PI} \mathrm{K}$ and $\mathrm{p}-\mathrm{AKT} / \mathrm{AKT}$ expression in HAECs of the ox-LDL group were significantly downregulated $(P<0.001)$. Moreover, compared with the miR106b NC + ox-LDL group, p-P13K/PI3K and p-AKT/AKT expression in the HAECs of the miR-106b mimics +oxLDL group were significantly up-regulated $(P<0.001)$.
Furthermore, compared with the miR-106b mimics + empty + ox-LDL group, the expressions of $\mathrm{p}-\mathrm{P} 13 \mathrm{~K} / \mathrm{PI} \mathrm{K}$ and $\mathrm{p}-\mathrm{AKT} / \mathrm{AKT}$ in the miR-106b mimics + PTEN + oxLDL group were significantly down-regulated $(P<0.001)$ (Figure 5).

\section{Discussion}

AS is an important cause of death and morbidity worldwide $(23,24)$. Although miRNAs participate in oxLDL-induced apoptosis of vascular endothelial cells (25), the detailed mechanism of miR-106b in AS is still unclear. 


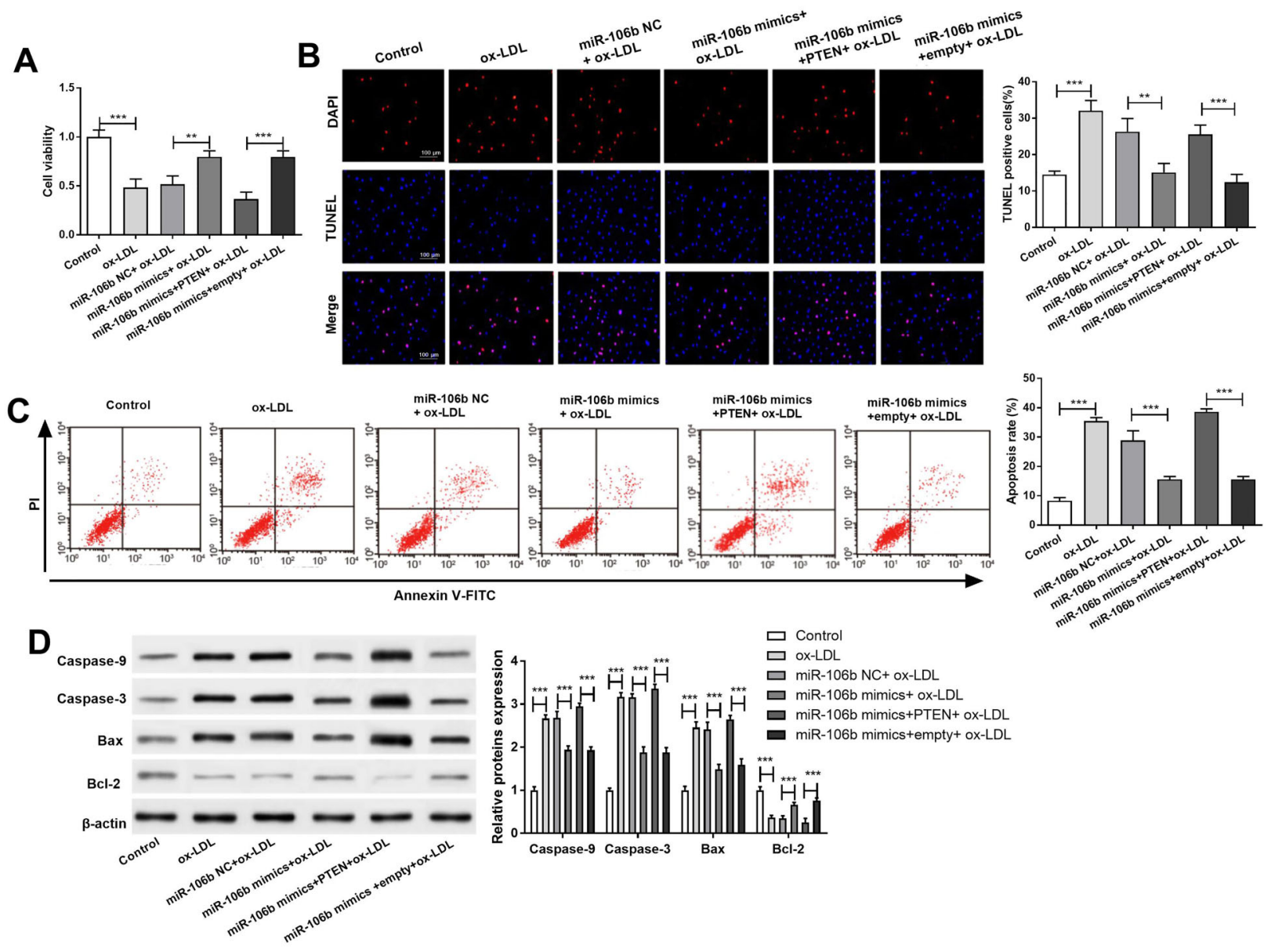

Figure 4. Overexpression of PTEN inhibited the anti-apoptotic effect of miR-106b. A, Proliferation of human aortic endothelial cells. B, Number of activity cells by TUNEL assay $(\times 400$, scale bar $100 \mu \mathrm{m})$. C, Apoptosis of human aortic endothelial cells. D, Expression of caspase-3, caspase-9, Bax, and Bcl-2 protein. Data are reported as means \pm SD. ${ }^{* *} \mathrm{P}<0.01$, ${ }^{* *} \mathrm{P}<0.001$ (ANOVA). ox-LDL: oxidizedlow-density lipoproteins; NC: negative control.

In this study, ox-LDL-induced HAECs were used to simulate the pathological state of atherosclerotic endothelial cells. The results showed that miR-106b was downexpressed in AS endothelial cells, and overexpression of miR-106b promoted proliferation and inhibited apoptosis of AS endothelial cells. Importantly, miR-106b activated the PI3K/AKT pathway by down-regulating the level of PTEN.

Endothelial cell apoptosis is the first step in the pathogenesis of AS (26). As an anti-apoptotic modulator, the down-regulation of miR-106b can inhibit proliferation and migration of renal cancer cells and induce apoptosis $(27,28)$. Li et al. (29) demonstrated that miR-106b-5p significantly enhances the expression level of Bcl-2 and reduces Bax expression. Importantly, members of the Bcl2 gene family regulate programmed cell apoptosis by controlling intracellular signaling promoting apoptosis and anti-apoptosis (30). However, a pervious study shows that overexpression of Bax accelerates apoptosis induced by cytokine deprivation in cell lines (31). Skała et al. (32) indicated that the high expression of pro-apoptotic Bax protein and low expression of anti-apoptotic $\mathrm{Bcl}-2$ protein are beneficial to inhibit cell proliferation and promote apoptosis. More importantly, $\mathrm{Bcl}-2$ protein also activates the downstream caspase cascade by regulating mitochondrial extracorporeal membrane permeabilization to perform apoptosis (33). Down-regulation of Bcl-2, procaspase-3, and pro-caspase- 9 can accelerate the proliferation of HAECs and promote endothelial cell apoptosis, thereby promoting the formation of AS (34). In fact, miR-106a mediates the caspase-3 pathway (35). The apoptotic rate and caspsse- 3 activity are significantly increased when the expression of miR-106b is inhibited (35). miR-106b prevents the apoptosis of endothelial cells in AS by blocking the activation of caspase-3 (36). In this study, the expression of Bax, caspase- 3 , and caspase- 9 in cells transfected with miR-106b was significantly 


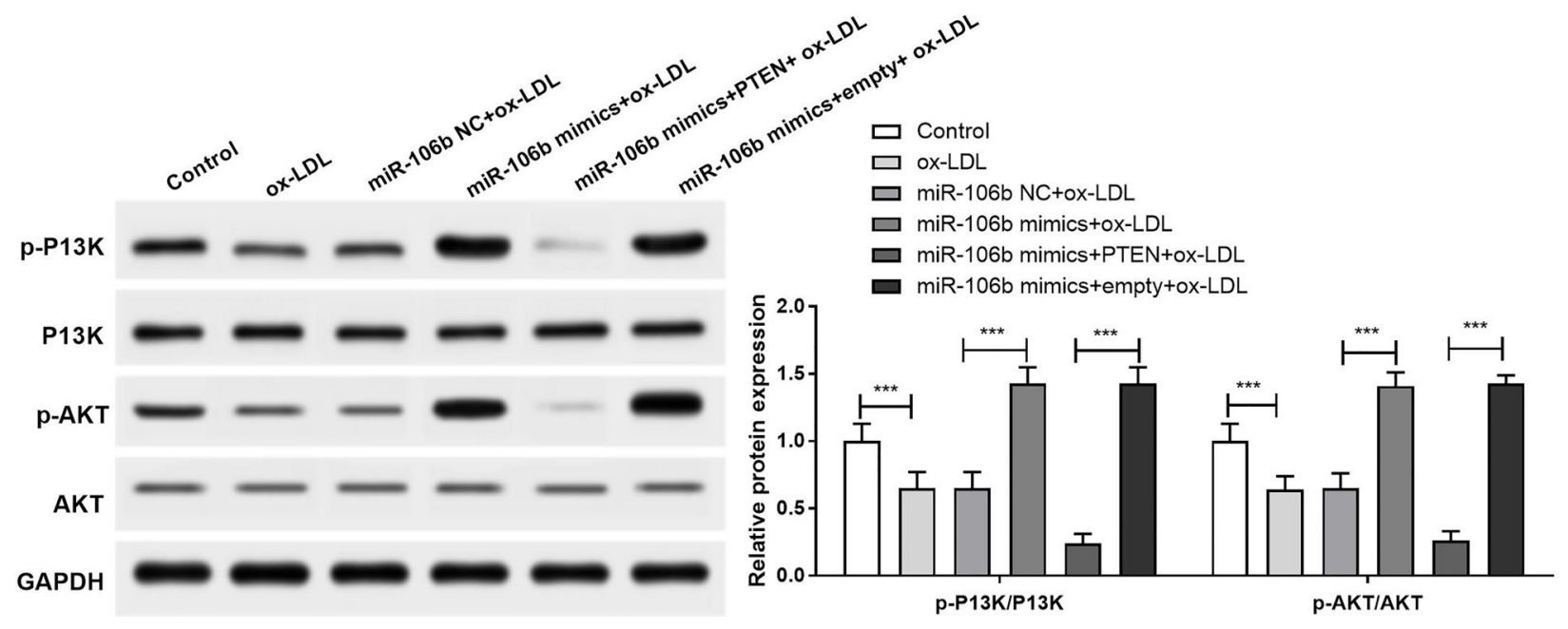

Figure 5. MiR-106b activated PI3K/AKT signaling pathway by down-regulating PTEN. Protein level of p-PI3K, total P13K, p-AKT, and total AKT was detected by western blot. Data are reported as means $\pm S D$. ${ }^{* \star *} P<0.001$ (ANOVA). ox-LDL: oxidized-low-density lipoproteins; NC: negative control.

decreased, while Bcl-2 was significantly up-regulated. Thus, we speculated that the overexpression of miR-106b in ox-LDL-induced AS inhibited endothelial cell apoptosis.

A previous study shows that the inhibition of miR-106b expression enhances the expression of PTEN (37). The up-regulation of PETN can induce endothelial cell dysfunction by attenuating the effectiveness and signaling of various angiogenic pathways in endothelial cells, which are involved in thrombosis of arteriovenous grafts (38). Moreover, overexpression of PTEN inhibits cell proliferation and increases the probability of apoptosis by inhibiting $\mathrm{Bcl}-2$ and promoting caspase-3 (39). As the downstream of PTEN, p-Akt is positively regulated by the overexpression of miR-106b (40). A previous study indicates that the inhibition of miR-106b expression enhances PTEN level, but inhibits the activity of downstream PI3K/AKT pathway (37). Meanwhile, inhibition of the PTEN expression level in

\section{References}

1. Tabas I, García-Cardeña G, Owens GK. The cell biology of disease: recent insights into the cellular biology of atherosclerosis. J Cell Biol 2015; 209: 13-22, doi: 10.1083/jcb. 201412052.

2. Suciu CF, Prete M, Ruscitti P, Favoino E, Giacomelli R, Perosa F. Oxidized low density lipoproteins: the bridge between atherosclerosis and autoimmunity. Possible implications in accelerated atherosclerosis and for immune intervention in autoimmune rheumatic disorders. Autoimmunity Rev 2018; 17: 366-375, doi: 10.1016/j.autrev.2017. 11.028.

3. Sahebkar A. Dual effect of curcumin in preventing atherosclerosis: the potential role of pro-oxidant-antioxidant mechanisms. Natural Product Res 2015; 29: 491-492, doi: 10.1080/14786419.2014.956212. vascular endothelial cells by miR-106b-5p prevents apoptosis of endothelial cells in AS (36). In the current study, the Targetcsan prediction and luciferase activity test showed that PTEN was a direct target of miR-106b. Meanwhile, western blot assay showed that overexpression of miR-106b inhibited endothelial cell apoptosis via the PTEN/P13K/AKT signaling pathway. Thus, we speculated that miR-106b might activate the PI3K/AKT pathway by down-regulating the expression of PTEN in ox-LDL induced HAECs.

In conclusion, overexpression of miR-106b in ox-LDLinduced AS inhibited endothelial cell apoptosis. Furthermore, miR-106b might activate the PI3K/AKT pathway by down-regulating the expression of PTEN in ox-LDLinduced HAECs. However, there are some limitations in this study including lack of clinical verification. Thus, future research is still needed.

4. Yuan J, Usman A, Das T, Patterson AJ, Gillard JH, Graves MJ. Imaging carotid atherosclerosis plaque ulceration: comparison of advanced imaging modalities and recent developments. AJNR Am J Neuroradiol 2017; 38: 664-671, doi: 10.3174/ajnr.A5026.

5. Rezvan A, Sur S, Jo H. Novel animal models of atherosclerosis. Biomed Engineering Lett 2015; 5: 181-187, doi: 10.1007/s13534-015-0200-4.

6. Micari A, Nerla R, Cremonesi A. Femoropopliteal atherosclerosis: do drug-eluting stents improve outcome? J Cardiovasc Med 2018; 19 Suppl 1: e91-e92, doi: 10.2459/ JCM.0000000000000593.

7. Shan DK, Zhou Y, Wang YX, Gao X, Wang W, Yang JJ, et al. Population-based cohort study in outcome of phased progression of atherosclerosis in China (PERSUADE): 
objective, rationale and design. J Geriatric Cardiol 2017; 14: 491-495, doi: 10.11909/j.issn.1671-5411.2017.08.001.

8. Feinberg MW, Moore KJ. MicroRNA regulation of atherosclerosis. Circ Res 2016; 118: 703-720, doi: 10.1161/ CIRCRESAHA.115.306300.

9. Ivanovska I, Ball AS, Diaz RL, Magnus JF, Miho K, Schelter $\mathrm{JM}$, et al. MicroRNAs in the miR-106b family regulate $\mathrm{p} 21 /$ CDKN1A and promote cell cycle progression. Mol Cell Biol 2008; 28: 2167-2174, doi: 10.1128/MCB.01977-07.

10. Li B, Shi XB, Nori D, Chao CK, Chen AM, Valicenti R, et al. Down-regulation of microRNA $106 \mathrm{~b}$ is involved in p21mediated cell cycle arrest in response to radiation in prostate cancer cells. Prostate 2011; 7: 567-574, doi: 10.1002/ pros. 21272 .

11. Xue B, Lu QY, Massie L, Qualls C, Mao JT. Grape seed procyanidin extract against lung cancer: the role of microrna106b, bioavailability, and bioactivity. Oncotarget 2018; 9: 15579-15590, doi: 10.18632/oncotarget.24528.

12. Park SH, Lee J, Yeo JS. On-chip plasmonic detection of microRNA-106a in gastric cancer using hybridized gold nanoparticles. Sensors \& Actuators B Chemical 2018; 262: 703-709, doi: 10.1016/j.snb.2018.02.010.

13. Ren J, Zhang J, Xu N, Han G, Geng Q, Song J, et al. signature of circulating microRNAs as potential biomarkers in vulnerable coronary artery disease. Plos One 2013; 8: e80738, doi: 10.1371/journal.pone.0080738.

14. Chen S, Chen X, Xiu YL, Sun KX, Zhao Y. Inhibition of ovarian epithelial carcinoma tumorigenesis and progression by microRNA $106 \mathrm{~b}$ mediated through the RhoC pathway. Plos One 2015; 10: e0125714, doi: 10.1371/journal.pone. 0125714.

15. Yan $\mathrm{H}, \mathrm{Ma} \mathrm{Y}, \mathrm{Li} \mathrm{Y}$, Zheng $\mathrm{X}$, Lv $\mathrm{P}$, Zhang $\mathrm{Y}$, et al. Insulin inhibits inflammation and promotes atherosclerotic plaque stability via PI3K-Akt pathway activation. Immunol Lett 2016; 170: 7-14, doi: 10.1016/j.imlet.2015.12.003.

16. Koide $\mathrm{M}$, Ikeda $\mathrm{K}$, Akakabe $\mathrm{Y}$, Kitamura $\mathrm{Y}$, Ueyama $\mathrm{T}$, Matoba $S$, et al. Apoptosis regulator through modulating IAP expression (ARIA) controls the PI3K/Akt pathway in endothelial and endothelial progenitor cells. Proc Nat Acad Sci USA 2011; 108: 9472-9477, doi: 10.1073/pnas.11012 96108.

17. Huang J, Kontos CD. PTEN modulates vascular endothelial growth factor-mediated signaling and angiogenic effects. J Biol Chem 2002; 277: 10760-10766, doi: 10.1074/jbc. M110219200.

18. Zhou K, Zhang T, Fan YD, Serick, Du G, Wu P, et al. MicroRNA-106b promotes pituitary tumor cell proliferation and invasion through PI3K/AKT signaling pathway by targeting PTEN. Tumour Biol 2016; 37: 13469-13477, doi: 10.1007/s13277-016-5155-2.

19. Shi DM, Bian $X Y$, Qin CD, Wu WZ. miR-106b-5p promotes stem cell-like properties of hepatocellular carcinoma cells by targeting PTEN via PI3K/Akt pathway. Onco Targets Ther 2018; 11: 571-585, doi: 10.2147/OTT.S152611.

20. Farber HW, Antonov AS, Romanov YA, Smirnov VN, Scarfo LM, Beer DJ. Cytokine secretion by human aortic endothelial cells is related to degree of atherosclerosis. Am J Physiol 1992; 262: H1088-H1095, doi: 10.1152/ajpheart.1992.262. 4.H1088.

21. Tang F, Yang TL, Zhang Z, Li XG, Zhong QQ, Zhao TT, et al. MicroRNA-21 suppresses ox-LDL-induced human aortic endothelial cells injuries in atherosclerosis through enhancement of autophagic flux: involvement in promotion of lysosomal function. Exp Cell Res 2017; 359: 374-383, doi: 10.1016/j.yexcr.2017.08.021

22. Livak KJ, Schmittgen TD. Analysis of Relative gene expression data using real-time quantitative PCR and the 2(-Delta Delta C(T)) method. Methods 2001; 25: 402-408, doi: 10.1006/meth.2001.1262.

23. Li Y, Yang C, Zhang L, Yang P. MicroRNA-210 induces endothelial cell apoptosis by directly targeting PDK1 in the setting of atherosclerosis. Cell Mol Biol Lett 2017; 22: 3, doi: 10.1186/s11658-017-0033-5.

24. Qin B, Shu Y, Long L, Li H, Men X, Feng L, et al. MicroRNA142-3p Induces atherosclerosis-associated endothelial cell apoptosis by directly targeting rictor. Cell Physiol Biochem 2018; 47: 1589-1603, doi: 10.1159/000490932.

25. Qin M, Luo Y, Meng XB, Wang M, Wang HW, Song SY, et al. Myricitrin attenuates endothelial cell apoptosis to prevent atherosclerosis: An insight into PI3K/Akt activation and STAT3 signaling pathways. Vascular Pharmacol 2015; 70: 23-34, doi: 10.1016/j.vph.2015.03.002.

26. Choy JC, Granville DJ, Hunt DW, Mcmanus BM. Endothelial cell apoptosis: biochemical characteristics and potential implications for atherosclerosis. J Mol Cell Cardiol 2001; 33: 1673-1690, doi: 10.1006/jmcc.2001.1419.

27. Liu Z, Yang D, Xie P, Ren G, Sun G, Zeng X, et al. MiR-106b and MiR-15b modulate apoptosis and angiogenesis in myocardial infarction. Cell Physiol Biochem 2012; 29: 851-862, doi: 10.1159/000258197.

28. Li Y, Chen D, Su Z, Liu J, Jin L, Shi M, et al. MicroRNA-106b functions as an oncogene in renal cell carcinoma by affecting cell proliferation, migration and apoptosis. Mol Med Rep 2016; 13: 1420-1426, doi: 10.3892/mmr.2015. 4656.

29. Li P, Shen M, Gao F, Wu J, Zhang J, Teng F, et al. An antagomir to microRNA-106b-5p ameliorates cerebral ischemia and reperfusion injury in rats via inhibiting apoptosis and oxidative stress. Mol Neurobiol 2016; 54: 2901-2921, doi: 10.1007/s12035-016-9842-1.

30. Ashkenazi A, Fairbrother WJ, Leverson JD, Souers AJ. From basic apoptosis discoveries to advanced selective BCL-2 family inhibitors. Nat Rev Drug Discov 2017; 16: 273-284, doi: 10.1038/nrd.2016.253.

31. Oltvai ZN, Milliman L, Korsmeyer SJ. Bcl-2 heterodimerizes in vivo with a conserved homolog, Bax, that accelerates programmed cell death. Cell 1993; 74: 609-619, doi: 10.1016/0092-8674(93)90509-O.

32. Skała E, Sitarek P, Toma M, Szemraj J, Radek M, Nieborowska-Skorska M, et al. Inhibition of human glioma cell proliferation by altered $\mathrm{Bax} / \mathrm{Bcl}-2-\mathrm{p} 53$ expression and apoptosis induction by Rhaponticum carthamoides extracts from transformed and normal roots. J Pharm Pharmacol 2016; 68: 1454-1464, doi: 10.1111/jphp.12619.

33. Zheng JH, Viacava Follis A, Kriwacki RW, Moldoveanu T. Discoveries and controversies in BCL-2 protein-mediated apoptosis. FEBS J 2016; 283: 2690-2700, doi: 10.1111/ febs.13527.

34. Li Y, Zhang K, Mao W. Inhibition of miR-34a prevents endothelial cell apoptosis by directly targeting HDAC1 in the setting of atherosclerosis. Mol Med Rep 2018; 17: 46454650, doi: $10.3892 / \mathrm{mmr} .2018 .8411$. 
35. Lu Z, Li S, Zhao S, Fa X. Upregulated miR-17 regulates hypoxia-mediated human pulmonary artery smooth muscle cell proliferation and apoptosis by targeting mitofusin 2. Med Sci Monit 2016; 22: 3301-3308, doi: 10.12659/MSM. 900487.

36. Zhang J, Li SF, Chen H, Song JX. MiR-106b-5p inhibits tumor necrosis factor- $\alpha$-induced apoptosis by targeting phosphatase and tensin homolog deleted on chromosome 10 in vascular endothelial cells. Chin Med J 2016; 129: 1406-1412, doi: 10.4103/0366-6999.183414.

37. Zheng Z, Zhang Y, Zhang Z, Yang Y, Song T. Effect of miR$106 \mathrm{~b}$ on invasiveness of pituitary adenoma via PTEN-PI3K/ AKT. Med Sci Monit 2017; 23: 1277-1285, doi: 10.12659/ MSM.900092.
38. Kuo HM, Lin CY, Lam HC, Lin PR, Chan HH, Tseng JC, et al. PTEN overexpression attenuates angiogenic processes of endothelial cells by blockade of endothelin-1/endothelin B receptor signaling. Atherosclerosis 2012; 221: 341-349, doi: 10.1016/j.atherosclerosis.2010.08.067.

39. Cheng ZY, Liang WT, Niu ZY, Xue F, Yao L. Effects of wild type PTEN gene on proliferation, apoptosis and the influence on apoptosis key factor $\mathrm{Bcl}-2$ and caspase family on K562 cells [in Chinese]. Sichuan Da Xue Xue Bao Yi Xue Ban 2009; 7: 679-683.

40. Zheng L, Zhang Y, Liu Y, Zhou M, Lu Y, Yuan L, et al. MiR$106 \mathrm{~b}$ induces cell radioresistance via the PTEN/PI3K/AKT pathways and p21 in colorectal cancer. J Transl Med 2015; 13: 252, doi: 10.1186/s12967-015-0592-z. 